\title{
Single Pulse Polarimetry of the Vela Pulsar
}

\author{
Jeffrey S. Kern \\ Department of Physics, New Mexico Institute of Mining and \\ Technology, Socorro NM 87801 USA : jkern@nrao.edu \\ Timothy H. Hankins \\ Department of Physics, New Mexico Institute of Mining and \\ Technology, Socorro NM 87801 USA : thankins@nrao.edu
}

Joanna M. Rankin

Physics Department, University of Vermont, Burlington VT 05405 USA rankin@physics.uvm.edu

\begin{abstract}
Multi-frequency, single-pulse polarimetric observations of the Vela pulsar have been made using the VLA. These high time resolution measurements allow us to probe the geometry, and the time dependent characteristics of the radio emission region. Evidence for multiple emission components with differing spectral indices is found.
\end{abstract}

\section{Introduction}

Although the Vela pulsar (B0833-45) is one of the few pulsars that astronomers know by name and is considered a prototypical pulsar by many; there is a surprising lack of observations of the pulsar at high radio frequencies.

The average pulse profile of Vela is known to evolve from type single below $1.4 \mathrm{GHz}$ (Hamilton et al. 1977) to resolved double at $4.8 \mathrm{GHz}$ (Gardner \& Whiteoak 1969). Krishnamohan \& Downs (1983) demonstrate that at $2.3 \mathrm{GHz}$ the radio emission region is well described as a composite of four components.

Our observations agree with the multiple component model, and show that the relative spectral development of these components continues above $4.8 \mathrm{GHz}$.

\section{Observations}

The Vela pulsar was observed u;sing the phased array mode of the VLA at three frequencies $(1.4,4.8$, and $8.4 \mathrm{GHz})$ during February 1998. The left and right circularly polarized signals were split into smaller channels (1-MHz at 1.4 $\mathrm{GHz}$, and $4-\mathrm{MHz}$ at 4.8 and $8.4 \mathrm{GHz}$ ), detected using a multiplying polarimeter, smoothed with a $200-\mu$ s time constant and then sampled at $213-\mu$ s resolution. The channels were calibrated independently, de-dispersed offline, and finally combined to form total power (Stokes I) measurements. 


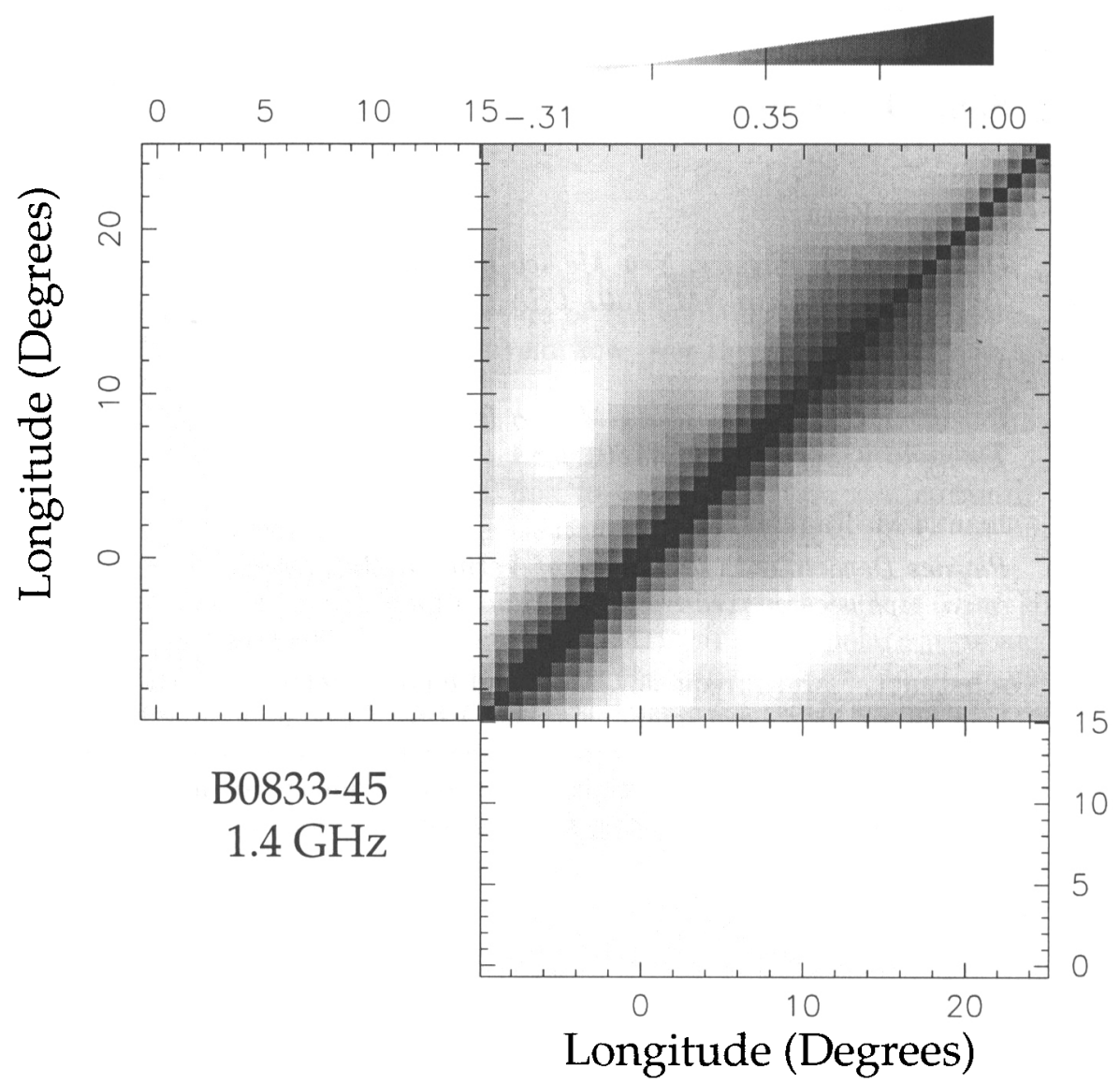

Figure 1. Longitude-longitude correlation map for B0833-45 at 1.4 $\mathrm{GHz}$.

\section{Time Dependent Behavior}

The primary advantage of recording single-pulse observations of pulsars is the ability to look at the time-dependent behavior of the pulsar. This provides important probes into the dynamics of the emission region. Figure 1 shows the longitude-longitude correlation of $\mathrm{B} 0833-45$ at $1.4 \mathrm{GHz}$. The diagonal line is the auto-correlation at zero lag and is, of course, one. The off-diagonal points represent zero lag cross-correlations between longitudes. The areas with high (dark) values correspond to regions which tend to fluctuate as a unit.

In this case there are obvious regions on the leading and trailing edge of the pulsar which are component like, and a region near $8^{\circ}$ which is anti-correlated with the leading edge. This suggests that there are at least three separate emission regions which fluctuate independently. 

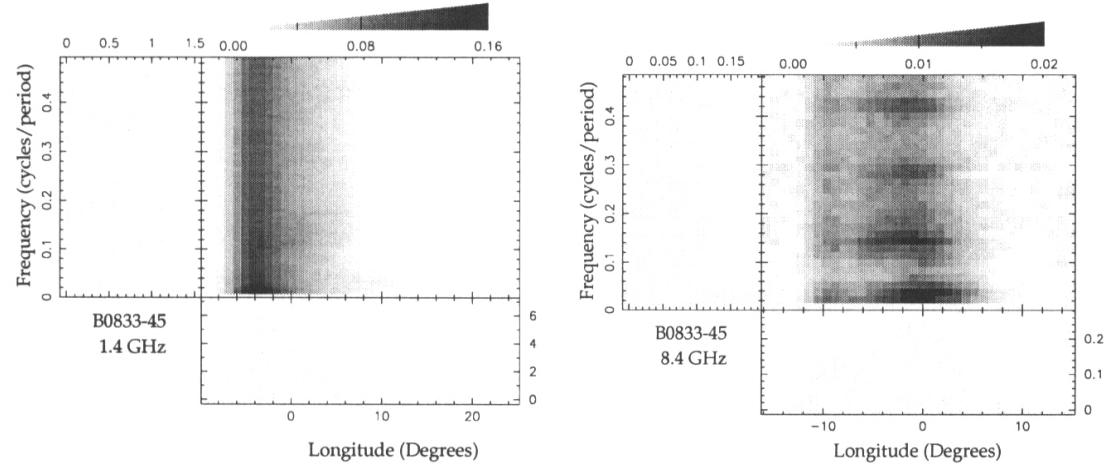

Figure 2. Longitude-resolved fluctuation maps of B0833-45 at 1.4 $\mathrm{GHz}$ (left) and $8.4 \mathrm{GHz}$ (right). Summed power in each longitude is plotted at the bottom of the figure, summed power in each frequency range is plotted along the left.

Figure 2 shows the longitude-resolved fluctuation spectra for Vela at 1.4 and $8.4 \mathrm{GHz}$. The zero-frequency component has been removed to show more clearly the fluctuations. Of particular importance is the difference in the spectra between the two frequencies. While the $1.4-\mathrm{GHz}$ profile has a relatively white fluctuation spectrum at all frequencies, the $8.4-\mathrm{GHz}$ data show significant spectral features associated with the trailing component. It is clear from the $8.4-\mathrm{GHz}$ map that there are at least two and probably three components contributing to the observed profile at this frequency.

\section{Average Profiles}

Using the measured positions of the fluctuation features at $1.4,4.8$ and $8.4 \mathrm{GHz}$, we find that the relative positions are consistent with the trailing component at $1.4 \mathrm{GHz}$ evolving to the second component in the $4.8 \mathrm{GHz}$ profile and eventually to the dominant feature at $8.4 \mathrm{GHz}$. Figure 3 shows the profiles aligned to reflect this correspondence, and clearly demonstrates the spectral evolution of the average profile.

\section{Conclusion}

Multi-frequency observations of B0833-45 show significant evolution with frequency. We find evidence for three fluctuating regions at $1.4,4.8$, and $8.4 \mathrm{GHz}$, suggesting emission components which persist over nearly a decade in frequency. In addition there is a broad non-fluctuating component in the profile, which agrees with Krishnamohan \& Downs (1983) description of four components. We find that the spectral index, and the fluctuation spectra of the components are different, which suggests differences in the underlying emission mechanism. 


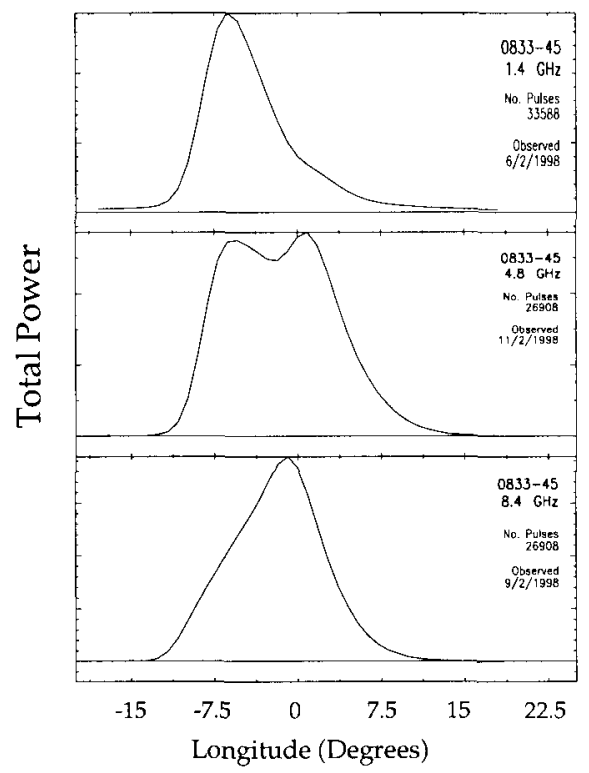

Figure 3. Average profiles of B0833-45 at $1.4,4.8$, and $8.4 \mathrm{GHz}$ respectively. The pulse phase has been adjusted to reflect the relative positions at different frequencies as described in the text.

\section{References}

Gardner, F. F., \& Whiteoak, J. B. 1969, Nature, 224, 891

Hamilton, P. A., McCulloch, P. M., Ables, J. G., \& Komesaroff, M. M. 1977, MNRAS, 180,1

Krishnamohan, S., \& Downs, G. S. 1983, ApJ, 265, 327 